NASA Contractor Report 191157

AIAA-93-2526

\title{
Three-Dimensional Numerical Simulation of Gradual Opening in a Wave Rotor Passage
}

Louis M. Larosiliere

Ohio Aerospace Institute

Brook Park, Ohio

Prepared for the

29th Joint Propulsion Conference and Exhibit

cosponsored by AIAA, SAE, ASME, and ASEE

Monterey, California, June 28-30, 1993 


\title{
THREE-DIMENSIONAL NUMERICAL SIMULATION OF GRADUAL OPENING IN A WAVE ROTOR PASSAGE
}

\author{
L. M. Larosiliere \\ Ohio Aerospace Institute \\ 22800 Cedar Point Road \\ Brook Park, OH 44142
}

\begin{abstract}
The evolution of the contact interface and the propagation of compression waves inside a single wave rotor passage gradually opening to and traversing an inlet port is studied numerically using an inviscid formulation of the governing equations. Insights into the response of the interface and kinematics of the flow field to various opening times are given. Since the opening time is inversely proportional to the rotational speed of the rotor, the effects of passage rotation such as centripetal and Coriolis accelerations are intrinsically coupled to the gradual opening process. Certain threedimensional features associated with the gradual opening process as a result of centripetal and Coriolis accelerations are illustrated. For the range of opening times or rotational speeds considered, a portion of the interface behaves like a vortex sheet that can degenerate into a complex interfacial structure. The vortices produced along the interface can serve as a stirring mechanism to promote local mixing. Coriolis and centripetal accelerations can introduce three-dimensional effects such as interfacial distortions in meridional planes and spanwise migration of fluid elements.
\end{abstract}

\section{NOMENCLATURE}

$\begin{array}{cl}\text { a } & \text { speed of sound } \\ \mathrm{B} & \text { dimensionless ratio of time scales } \\ \hat{\mathrm{e}} & \text { unit vector } \\ \mathrm{E} & \text { total energy per unit mass } \\ \mathrm{h}_{\mathrm{tR}} & \text { rothalpy } \\ \mathrm{L} & \text { passage length } \\ \mathrm{Mo}, \mathrm{M}_{\Omega} & \text { wheel Mach number } \\ \hat{n} & \text { unit normal vector } \\ \mathrm{p} & \text { pressure } \\ \mathrm{R}_{\Omega} & \text { rotation parameter }\end{array}$

$\begin{array}{ll}R_{H} & \text { hub radius } \\ R_{T} & \text { tip radius } \\ T & \text { temperature } \\ t & \text { time } \\ \tau & \text { reference time scale } \\ \gamma & \begin{array}{l}\text { relative velocity vector } \\ \text { ratio of specific heats } \\ \rho\end{array} \quad \begin{array}{l}\text { density } \\ \theta\end{array} \\ \theta & \begin{array}{l}\text { azimuthal coordinate in a } \\ \text { stationary reference frame } \\ \text { azimuthal coordinate on the } \\ \text { rotating passage } \\ \text { passage sector angle } \\ \text { rotational speed }\end{array} \\ \end{array}$

\section{Subscripts}

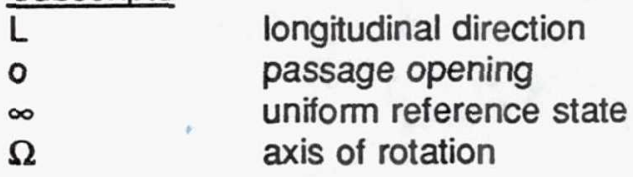

\section{INTRODUCTION}

There is strong impetus for increasing the maximum cycle temperature of gas turbine engines in order to achieve higher cycle efficiencies. A wave rotor augmented cycle provides the potential for this increased performance within the limits of current materials technology [1]. Conceptually, the wave rotor is based on the idea of energy exchange using unsteady waves. A wave rotor cycle can play the role of a mechanically coupled compressor and turbine, delivering work to compress one gas stream while extracting work from another expanding gas stream. Since both expansion and compression are performed cyclicly in the rotor, the allowable material thermal limits are usually not exceeded.

A wave rotor (Fig. 1) typically consists of 
a number of cylindrical passages mounted along the periphery of a rotating drum. Each of these passages is cyclicly exposed to different end conditions that generate unsteady waves for energy transfer. As a wave rotor passage rotates into a stationary inlet-port, the passage is gradually exposed to the port flow. This process is called gradual opening and the period of adjustment wherein the leading and trailing walls of a rotor passage move out of a solid endwall allowing the passage to be fully exposed to the inlet-port conditions is herein referred to as the opening time.

Eidelman [2] presented a numerical simulation of gradual opening in wave rotor passages showing that the dynamics of the passage opening significantly impact the flow pattern. The gradual opening process results in a significant distortion (i.e., obliquity) of the fluid interface in the passage-to-passage plane and also leads to a finite time for the formation of the shock. These events can adversely affect the performance of a wave rotor. Since Eidelman's model was two-dimensional it could not include the effects of Coriolis and centripetal accelerations brought on by the rotation of the passage.

For a given rotor passage geometry, the gradual opening time is inversely proportional to the rotational speed of the passage. Hence, the problem of gradual opening is intrinsically coupled to that of rotation. Larosiliere and Mawid [3] have shown that centrifugal and Coriolis accelerations brought on by the rotation of a channel can significantly impact unsteady gasdynamic wave processes. Therefore, the response of the flow in a wave rotor passage to the gradual opening process may be altered by Coriolis and centripetal accelerations resulting from the rotation of the passage.

In the present study, the impact of gradual opening on the flow pattern in a wave rotor passage is re-examined in order to illustrate certain features due to Coriolis and centripetal accelerations that have not heretofore received much attention. A three-dimensional Euler model of the gradual opening problem is formulated in a rotating reference frame for a single wave rotor passage. The equations are numerically solved in order to track the gradual opening transient and the subsequent flow development as the passage traverses the inlet port. Variations in the response of the flow field in a passage of fixed geometry are examined for a wide range of rotational speeds. A discussion of the results highlighting implications for wave rotor design is provided.

\section{FORMULATION}

The gradual opening problem involves a coupling between the inlet-port flow and the flow in the wave rotor passages. Flow through the port is for the most part steady [1] , but the flow in the wave rotor passages is unsteady and periodic in time. This periodicity is a consequence of the cyclic nature of the wave rotor processes. Interaction of the port flow with that in the passages during the gradual opening time may generate a local unsteady flow in the inlet port. This is probably a small scale unsteadiness since the width of the inlet port is usually much larger than that of a single passage.

There are currently two different approaches for treating the gradual opening problem in a wave rotor. First, the more general approach adopted by Welch [4] involves the determination of a time-periodic flow field whose physical domain includes the inlet port and all of the passages spanning that port. The second approach called the Signal-Storage-andReconstruction (SSR) technique, introduced by Erdos et al. [5], determines the entire flow field by considering only the port and a single passage. The time periodicity condition is explicitly used to construct the flow in the other passages. Both techniques have been applied in rotor/stator interaction studies and are known to be computationally intensive in three-dimensions.

In the present study, the aim is not to provide a detailed description of the coupling between the inlet-port flow and the flow in the rotor passage, but rather to characterize the response of the flow field in a rotating passage to the gradual application of the stationary inlet-port conditions. Thus, a single wave rotor passage is tracked as it passes stationary endwalls and the 
inlet port. The flow in the inlet port is not calculated; however, its effect is accounted for through boundary conditions at the inflow plane.

\section{Governing Equations}

The geometric configuration chosen to simulate the gradual opening problem is illustrated in Figure 2. It consists of a single cylindrical wave rotor passage of length $L$, and sector angle $\Phi$. At $t=0$ the passage is fully enclosed. Subsequent to this time, the solid-wall boundary conditions at the left side $(x=0)$ of the passage are gradually replaced with those of the inflow-plane as the passage rotates out of the endwall. The right side is always closed. Viscous effects and heat conduction are neglected.

The geometry is made dimensionless by scaling the axial coordinate with the passage length $L$, the circumferential coordinate with the passage sector angle $\Phi$, and the radial coordinate with the tip radius $R_{T}$. Let $\left(R, x, \theta^{\theta}\right)$ denote a dimensionless cylindrical coordinate system attached to the rotor. The $x$-axis lies along the axis of rotation and the rotating channel is restricted by the five walls: $\theta^{d}=0, \theta^{l}=1, R=$ $R_{H} / R_{T}, R=1$, and $x=1$. The equations are made dimensionless by introducing a reference length $L_{\text {ro }}$ for the gradient operator, a reference time $\tau$, a reference density $\rho_{\infty}$, and a reference speed of sound $a_{\infty}$. As there is as yet no obvious way to assign definite values to the reference quantities, they will be left unspecified for now.

Combinations of the reference scales are selected so that the following quantities, defined without asterisks, are dimensionless:

$$
\begin{aligned}
& t=\frac{t^{*}}{\tau}, p=\frac{\rho^{*}}{\rho_{-}}, \phi=\frac{W^{*}}{a_{-}}, p=\frac{p^{*}}{\rho_{-} a^{2}}, E=\frac{E^{*}}{a^{2}} \\
& x=\frac{x^{*}}{L}, R=\frac{R^{*}}{R_{T}}, \theta=\frac{\theta^{*}}{\Phi}, \vec{\nabla}=L_{\text {rot }} \vec{\nabla}^{*} .
\end{aligned}
$$

The flow is modelled by the unsteady threedimensional Euler equations [6] written in a rotating reference frame,

$$
B \frac{\partial p}{\partial t}+\vec{\nabla} \cdot(p w)=0
$$

$$
\begin{array}{r}
B \frac{\partial \rho W}{\partial t}+\nabla \cdot(\rho \omega)+2 R_{Q} \rho \hat{\theta}_{Q} \times- \\
M_{\alpha}^{2} \rho \nabla\left(\frac{R^{2}}{2}\right)=-\nabla p
\end{array}
$$

$$
B \frac{\partial \rho E}{\partial t}+\vec{\nabla} \cdot\left(\rho \Phi h_{\omega A}\right)=0
$$

completed by the perfect gas state relation,

$$
T=\gamma \frac{p}{p}
$$

where $h_{\mathrm{LR}}$ is the rothalpy,

$$
h_{B P}=\frac{T}{\gamma-1}+\frac{1}{2} W^{2}-\frac{1}{2} M_{0}^{2} R^{2},
$$

and $E$ is the total energy per unit mass in the rotating frame,

$$
E=h_{t R}-\frac{p}{\rho}
$$

In addition to the ratios of geometric length scales (i.e., $\Phi R_{T}: R_{T}: L: L_{\text {ref }}$ ) implicitly contained in the gradient operator, the equations of motion depend on three other dimensionless parameters

$$
B=\frac{L_{\text {red }} / a_{\text {O }}}{\tau}, M_{\mathrm{Q}}=\frac{\Omega R_{T}}{a_{\mathrm{C}}}, R_{\mathrm{Q}}=\frac{L_{\text {red }} / \Omega_{\mathrm{O}}}{1 / \Omega} .
$$

A proper scaling of the problem is required in order that the relative magnitude of each term in 
the equations of motion is indicated by a dimensionless factor preceding that term. Note that the wheel Mach number $M_{\Omega}$ already indicates the magnitude of the centrifugal force but $B$ and $R_{\Omega}$ demand further insights into the character of the problem. Boundary and initial conditions of the flow may be interrogated to define intrinsic reference scales of the problem.

\section{Boundary Conditions}

Boundary conditions at $\mathrm{x}=0$ are formulated by introducing a gate function $G(\theta)$ simulating the inlet-port opening and closing. An expression for the gate function is given as,

$$
G(\theta)=H(\theta-\Phi)-H\left(\theta-\theta_{p}\right)
$$

where $H(\theta)$ is Heavyside's unit step function, the angle $\theta$ is an absolute angle measured in the frame of the stationary port, and $\theta_{p}$ is the angular position of the port closing. A transformation from the absolute frame $(\theta)$ to the rotating frame $\left(\theta^{n}\right)$ can be written as,

$$
\theta=\theta^{\prime}+\frac{\tau}{\tau_{0}} t-\frac{2 \pi}{\Phi} k \quad 0 \leq \theta^{\prime} \leq 1, k=0,1, \ldots
$$

where $\tau_{0}=\Phi / \Omega$ is the passage opening time. The above expression assumes that the origin of the two reference frames coincide at $t=0$. Introducing this transformation into equation (1g) gives an indication of whether or not a specific spatial location on the rotor passage at $\mathrm{x}=0$ corresponds to a solid endwall ( $G\left(\theta^{\circ}\right)=0$ ) or to the inflowplane ( $G\left(\theta^{\prime}\right)=1$ ) at a given time. Inflow boundary conditions are discussed in the computational solution section. Free-slip wall velocity boundary conditions are:

$$
\mathbf{W} \cdot \hat{n}=0 \text { for },\left[\begin{array}{c}
R=\frac{R_{H}}{R_{T}}, R=1 \\
x=0, x=1 \\
\theta^{\prime}=0, \theta^{\prime}=1
\end{array}\right]
$$

\section{Initial Conditions}

At $t=0$, the passage is assumed to contain a gas at rest ( $w=0$ ) relative to the closed passage walls. Uniform thermodynamic conditions are prescribed (i.e., $p=1 / \gamma, \rho=1$ ). Although a radial variation of the thermodynamic properties can be prescribed by assuming homentropic solid-body rotation, this is probably not necessary for the range of parameters to be considered. Nevertheless, the present initialization procedure is thought to be close to the conditions in an actual rotor where the gas has been brought to rest through wave actions.

\section{Scaling}

For the sake of simplicity, the uniform thermodynamic reference state " $\infty$ " is taken to be that which exists in the passage at the initial time $(t=0)$. In determining the reference length scale for the gradient operator and the reference time scale, attention should be focused on the region where and over what time period the dependent variables are changing most rapidly. It is anticipated that during the gradual opening period, most of the changes in the flow field will occur over a distance on the order of the passage width. This implies that

$$
B=\frac{\Phi R_{T} / a_{\alpha}}{\tau_{0}}=M_{Q}, R_{Q}=\Phi M_{Q}, t \leq 1
$$

Thus, during the gradual opening period, the wheel Mach number $M_{\Omega}$ is the time constant of the unsteady effects. That is, the higher the wheel Mach number, the more rapid will be the adjustment of the flow field in the passage to the application of the inflow conditions.

Subsequent to the gradual opening period, it is anticipated that the length of the passage is the intrinsic length scale over which rapid changes will occur. Employing the longitudinal transport time $\tau_{\mathrm{L}}=\nu \mathrm{a}_{\infty}$ as the intrinsic time scale results in the following dimensionless parameters 


$$
B=\frac{L / a_{\infty}}{\tau_{L}}=1, R_{0}=\left(L / R_{t}\right) M_{0}, t>\frac{\tau_{0}}{\tau_{L}}
$$

Hence, subsequent to the gradual opening period, unsteady and convective effects are of the same order of magnitude.

It should be emphasized that the choice of intrinsic length and time scales depends on the parameter range under consideration. Observe that during the gradual opening period (i.e., physical times less than or equal to the opening time), a scaling is introduced which establishes $M_{\Omega}$ as the dimensionless parameter indicating the relative order of magnitude of unsteady effects. However, subsequent to the gradual opening period, a different scaling, correctly indicating that unsteady and convective effects are of the same order of magnitude, should be adopted.

For fixed geometry and boundary conditions, the governing equations are characterized by only one parameter, the wheel Mach number $M_{\Omega}$. The wheel Mach number has three different interpretations. First, it gives a measure of the relative strength of the centripetal acceleration. Second, during the gradual opening transient, the wheel Mach number can also be interpreted in terms of the ratio of the wave transport time across the width of the passage $\left(\Phi R_{T} / a_{\infty}\right)$ to the passage opening time. Hence, for a fixed geometry and inlet conditions, increasing the wheel Mach number reduces the opening time relative to the wave transport time across the passage width. This implies a smaller distortion (i.e., relative to a planar orientation) of the wave fronts and also a reduction of the number of wave reflections. Third, $M_{\Omega}$ gives a measure of the deformation of the interface in terms of the ratio of tangential $(\sim t)$ to longitudinal $\left(\sim t / M_{0}\right)$ extent of the interface during the gradual opening period. Moreover, the effects of Coriolis acceleration on the fluid contact interface will tend to be more pronounced due to a larger $R_{\Omega}$ with increasing wheel Mach number.

\section{Computational Scheme}

The governing equations are solved using a four stage Runge-Kutta algorithm developed by Chima and Yokota [7]. This scheme employs explicit time-marching along with central-difference spatial discretization on a boundary-fitted $\mathrm{H}$-mesh. The local truncation error is formally second-order both in space and time. Second and fourth difference artificial dissipation terms are used to capture discontinuities and to control non-linear instabilities. Further details concerning implementation of this scheme can be found in Reference 7. The treatment of the inflow boundary follows.

The base flow at the inflow-plane $(x=0)$ can be expressed as the sum of two components: a steady flow and an unsteady perturbation. This flow is assumed to evolve from the steady inlet-port flow, which is partially specified and partially computed from the solution interior to the rotor passage. It is this interior solution which is responsible for the unsteady perturbation. In addition, the base flow is assumed to be isentropic. The procedure adopted for describing the base flow at the inflow-plane consists of prescribing the incoming waves and using characteristic equations to supply the outgoing waves (see for example, Thompson [8] for more details).

At each point on the inflow boundary four pieces of information must be furnished in order to calculate the flow variables. Assuming that the upstream axial Mach number is always subsonic, the Riemann invariant along the downstream running characteristic $\left(J^{+}=w_{x}+\frac{2 a}{\gamma-1}\right)$, the relative tangential velocity, the radial velocity, and the upstream entropy are specified. The Riemann invariant along the upstream running characteristic $\left(J^{-}=w_{x}-\frac{2 a}{\gamma-1}\right)$ is extrapolated from the interior. This procedure allows all of the flow variables to be updated along the inflow boundary. 
TABLE I.- SIMULATION PARAMETERS

\begin{tabular}{||c|c|c|c||}
\hline$M_{\Omega}, M_{0}$ & $\tau_{d} \tau_{L}$ & $R_{\Omega}$ (based on $R_{T} \Phi$ ) & $R_{\Omega}$ (based on $L$ ) \\
\hline 0.1 & 0.573 & 0.009 & 0.150 \\
\hline 0.2 & 0.287 & 0.017 & 0.300 \\
\hline 0.3 & 0.191 & 0.026 & 0.450 \\
\hline 0.4 & 0.144 & 0.034 & 0.600 \\
\hline 0.6 & 0.096 & 0.052 & 0.900 \\
\hline
\end{tabular}

\section{RESULTS AND DISCUSSION}

Five cases are presented to illustrate the flow dynamics of the gradual opening process and also the subsequent development of the interface during the time period for a single passage to traverse across an inlet port having an angular extent of approximately 13 passage widths (i.e., $\theta_{p}=14.2$ ). A fixed passage geometry with a length-to-width ratio $L\left(R_{T} \Phi\right)=17.4$, a hubto-tip radius ratio $R_{H} / R_{T}=0.934$, and a length-totip radius ratio $L / R_{T}=1.5$ was used. This geometry reflects the NASA wave rotor experimental rig [9]. The uniform computational mesh consisted of 300 longitudinal nodes, 30 radial nodes, and 30 circumferential nodes. A $\mathrm{CFL}=0.5$ was used to adequately resolve the gradual opening transient.

Uniform inflow-plane conditions consisting of a downstream running Riemann invariant $\mathrm{J}^{+}=$ 7.08, upstream entropy $p_{d} / T_{0}^{\gamma(r-1)}=0.126$, and zero circumferential and radial relative velocity components $\left(w_{\theta}=0, w_{R}=0\right)$ were assumed. These conditions are typical for a wave rotor passage filled with a quiescent charge of air at ambient conditions and encountering a perfectly matched (i.e., zero incidence to the rotor) inlet port supplying hot, high-pressure driver gas. The inflow-plane conditions are held fixed along with a specific heat ratio $\gamma=1.4$. The range of simulation parameters are given in Table I. Also listed in Table I are the ratios of full passage opening time $\left(\tau_{0}\right)$ to longitudinal transport time $\left(\tau_{L}\right)$.

\section{Gradual Opening Transient Response}

The transient response of the flow field in the passage to the gradual opening process is illustrated in Figures 3,4 , and 5 . These figures show pressure contours (Fig. 3), entropy contours (Fig. 4), and velocity vectors (Fig. 5 ) in a passage-to-passage plane (i.e., $x-\theta^{*}$ ) at midheight for several wheel Mach numbers and other parameters as given in Table I. Two instants during the gradual opening transient are presented, specifically the times when the passage is half opened (Fig. 3(a), 4(a), 5(a)) and fully opened (Fig. 3(b), 4(b), 5(b)).

As the passage gradually opens, a series of curved pressure waves followed by a contact interface, as shown in Figures 3(a) and 4(a), emanate from the small opening. The waves penetrate into the passage and reflect from the trailing wall to interact with other incoming pressure waves and with the interface. Interaction of the reflected pressure waves with the interface produces baroclinic vorticity (i.e., $\vec{\nabla} p \times \vec{\nabla} p$ ) at the interface as is clearly evident in Figure 5. For Mo $=0.1$ and 0.2 , a large clockwise-oriented vortex is observed. A portion of the interface wraps around this vortex and assumes a spiral configuration. In this spiralling portion of the interface, the density gradient across the interface combines with the vortex-induced radialpressure gradient (Fig. 3 ) to generate additional vorticity, possibly of opposite rotation.

Note that the pressure waves undergo multiple reflections (Fig. 3 ) from both the trailing and leading walls of the passage. As the wheel Mach number is increased, a smaller number of 
wave reflections occurs due to the more rapid adjustment of the flow. Thus, for increasing wheel Mach number during the gradual opening period, the baroclinic vorticity production at the interface due to interaction with reflected waves is reduced as is evident in Figure 5.

It is observed from Figure 4 that the interface evolution during the gradual opening period exhibits increasing distortion. The interface distortion is caused by two mechanisms: (1) deformation of fluid elements comprising the interface as a result of gradual opening of the passage and (2) generation of vorticity (i.e., local rotation of fluid elements) by the actions of baroclinic torques, Coriolis forces, and vortex stretching. If the opening time were instantaneous, the interface would be a planar surface normal to the leading and trailing walls of the passage, but due to the finite opening-time, the interface deforms.

The higher the wheel Mach number, the smaller the deformation as previously discussed. For $M o=0.1$, a portion of the interface acquires a quasi-planar orientation (Fig. 4) extending across the passage width similar to that with instantaneous opening, followed by a trailing portion having a spiral configuration. The orientation of this spiral is consistent with the sign of baroclinic vorticity production (Fig. 5) due to wave reflections from the trailing wall. A similar but less developed interfacial structure is observed for $\mathrm{Mo}=0.2$. In comparison, the $\mathrm{Mo}=$ 0.3-0.4 cases experience a much smaller deformation with no evidence of a spiral configuration. It can be inferred from the above considerations that whether the maximum transient response (i.e., the creation of a quasiplanar interface across the entire passage width) and the generation of a spiral configuration occur during or after the gradual opening period depends on the wheel Mach number.

Since $R_{\Omega}$ (based on the passage width) is small (Table I) for the cases considered, the effect of Coriolis forces is negligible during the gradual opening period. Hence, the flow is mostly two-dimensional during this time period which implies that vortex stretching and tilting is small.
Two-Dimensional Temporal Evolution

The evolution of the interface and the propagation of compression or shock waves are shown in Figures 6(a)-(e) through density contours in a passage-to-passage plane at midheight for wheel Mach numbers $M o=0.1,0.2$, $0.3,0.4,0.6$. Note that the dimensionless times are based on the longitudinal transport time scale $\left(\tau_{L}\right)$ and the reference length is taken as the passage length $L$. Figures $6(a)-(e)$ spans the time period required for the passage to traverse the width of the inlet port.

The plots suggest an interpretation of the interface in terms of two distinct structures: (1) a quasi-planar interface and (2) a trailing spiral interface resembling an evolving vortex sheet. The characteristics of the interface evolution depend on two parameters: the wheel Mach number and $R_{\alpha}$ based on the passage length. For Mo $=0.1-0.2$ (Figs. 6(a)-(b)), the spiraling interface undergoes substantial distortion and eventually degenerates into a complex configuration $(t=1 \cdot 0-2.0)$. The quasi-planar interface exhibits a much smaller distortion with a slight bulge indicating a possible break-away from the spiral interface.

For Mo $=0.4-0.6$ (Figs. $6(\mathrm{~d})-(\mathrm{e}))$, the spiral interface is smaller and better defined. The quasi-planar interface is more oblique and at $t=$ 1.33 , the interface appears to be spreading along both the trailing and leading walls. This is due to Coriolis and centripetal accelerations as will be shown later. For $\mathrm{Mo}=0.3$ (Fig. 6(c)) the interface orientation is intermediary to the $\mathrm{Mo}=0.1$ and $\mathrm{Mo}$ $=0.6$ cases.

Also of interest in Figs. 6(a)-(e) is the formation of the shock wave and its interaction with the interface. It is apparent that at low wheel Mach numbers or large opening times, a relatively long time period is required for the formation of the shock wave. Figure 6(a) shows a series of compression waves propagating along the entire length of the passage for $\mathrm{Mo}=0.1$. Some of these waves reflect from the back wall and subsequently coalesce with part of the remaining incident compression waves to form a weak shock $(t=1.33)$. At a wheel Mach number of Mo $=0.6$ (Fig. 6(e)), the shock is formed in a relatively short time period $(t=0.333)$. 
The interaction of the shock with the interface is exhibited between $t=1.667$ and $t=$ 2.0. For $M o=0.1$, this interaction generates two distinct mixing pockets and at the same time, stretches the quasi-planar interface. Note also that the interaction of the shock with the interface spawns a transmitted shock along with a series of reflected pressure waves. An interesting result of the interface/reflected shock interaction is the counter-clockwise rotation of the quasi-planar interface for $\mathrm{Mo}=0.1-0.3$, whereas for $\mathrm{Mo}=0.4$ 0.6 , a complex interfacial pattern develops.

Further insight into the evolution of the interface is given in Figure 7 which shows contours of the radial component of vorticity in a passage-to-passage plane at mid-height for Mo $=0.1,0.3,0.6$ at $t=0.667,1.33$, and 2.0. A negative vorticity implies a clockwise rotation. The vorticity distributions are consistent with the interface orientations in Figs. 6 (a)-(e). For Mo = 0.1 at $\mathrm{t}=0.667$ (Fig. $7(\mathrm{a})$ ), the negative vorticity, generated by wave reflections from the trailing wall, appears to be surrounded by a region of positive (counter-clockwise) vorticity, possibly generated by wave reflections from the leading wall. There is a local positive leading-wall vortex for $\mathrm{Mo}=0.6$ at $\mathrm{t}=0.667$. Also, at $\mathrm{t}=1.33$ for $\mathrm{Mo}$ $=0.6$, the negative spiral vortex is sandwiched between positive vortices at both walls.

Interaction of the reflected shock with the interface results in a complex vorticity distribution as is evident in Fig. 7 at $t=2.0$. Multiple pockets of negative and positive radial vorticity are generated for $M o=0.1$. For $M o=0.6$, the negative spiral vorticity is not discernible.

\section{Three-Dimensional Effects of Passage Rotation}

The three-dimensionality of the interface development is illustrated in Figure 8 at two instants subsequent to the gradual opening transient. Entropy contours on passage-topassage planes at hub, mean, and tip radii are shown for wheel Mach numbers $\mathrm{Mo}=0.1,0.3$, 0.6 .

For $\mathrm{Mo}=0.1$, there does not appear to be any substantial three-dimensionality in the interface configuration. Similar patterns can be found at hub, mean, and tip. However, for Mo = 0.3 , there is more intense mixing at the tip spiral vortex relative to the hub. The center of the spiral vortex is at roughly the same axial position for hub, mean, and tip sections. For $M o=0.6$, a radial distortion of the interface with driven fluid (lower entropy) on top and driver fluid (higher entropy) on the bottom is observed. This is a result of baroclinic vorticity generation by the centripetal acceleration at the interface.

For Mo $=0.6$, there exists circulatory flows normal to the mean flow due to vorticity generation by Coriolis forces. This is demonstrated by the presence of interfacial material along the trailing and leading walls of the passage at mid-height for $\mathrm{Mo}=0.6$. Material from hub and tip migrate spanwise towards midheight.

\section{Wave Rotor Design Implications}

The static pressure and axial velocity distribution along the passage length at midheight and mid-passage are shown in Figure 9 for $t=0.667$. This figure gives a global perspective of the effects of various gradual opening times. Also included in Figure 9 is the instantaneous opening case as a reference. It can be seen that reducing the gradual opening time (i.e., increasing $M_{\Omega}$ ) for a fixed geometry allows a more rapid shock formation and propagation. The time for shock formation is significantly greater than that with instantaneous passage opening. This has strong implications for port timing and wave arrangement.

Increasing the opening time (i.e., reducing $M_{\alpha}$ ) generates substantial blockage near the interface resulting in a locally higher throughflow velocity at a reduced static pressure. This is a loss mechanism (i.e., mixing loss between driver and driven gas) that should be addressed by wave rotor designers. The flow blockage is caused by the vortical structures that are generated by the gradual opening process and sustained by the action of centripetal and Coriolis accelerations at the interface. For a fixed overall geometry, it may be possible to reduce this blockage by profiling the passage walls near the inlet. This profiling needs to be performed in a three-dimensional fashion if the adverse effects of the centripetal and Coriolis accelerations are to be countered. It should also be noted that an optimum wheel Mach number allowing a 
compromise between the two-dimensional adverse effects of gradual opening and the associated three-dimensional effects resulting from Coriolis and centripetal accelerations may be found.

\section{CONCLUSIONS}

A numerical simulation of the flow dynamics of gradual opening of a wave rotor passage shows that the flow development is complex and three-dimensional. For fixed geometry and boundary conditions, the response of the flow in the rotor passages depends on the wheel Mach number. At low wheel Mach numbers (i.e., Mo < 0.3 ) or long opening times, the interface between in-flowing driver gas and the driven gas in the passage becomes very distorted due to interactions with multiple reflected compression waves from the trailing and leading walls of the passage. Vortices are produced at the interface and it is observed that these vortices can serve as a stirring mechanism to promote mixing between driver and driven fluid along the interface. For higher wheel Mach numbers (i.e., Mo $>0.2$ ) or short opening times, the amount of compression wave reflection is reduced which results in a smaller distortion of the interface due to interactions with reflected compression waves. However, the higher centripetal and Coriolis accelerations generate additional vorticity which causes a threedimensional interface distortion.

The development of the wave system is also dependent on the wheel Mach number or passage opening time. For low wheel Mach numbers or longer opening times, a substantial time period is required for the formation of shocks. Due to the highly distorted interface, subsequent interaction of the reflected shock with the interface results in the creation of a complex flow pattern.

It can be concluded that the gradual opening process and the influence of rotation on the flow patterns are intimately coupled. This should be kept in mind during the wave rotor design process, not only for proper port-timing and wave arrangement but also because of the losses which will occur due to mixing and wave reflections. Rotating faster in order to reduce the adverse effects of gradual opening may create other adverse effects due to Coriolis and centripetal accelerations. The results presented herein and additional results with the inclusion of viscous forces may help guide the designer in achieving an optimum compromise.

\section{REFERENCES}

1. Shreeve, R.P. and Mathur, A. (eds.): Proceedings of the 1985 ONR/NAVAIR Wave Rotor Research and Technology Workshop. NPS67-85-008.

2. Eidelman, S.: "The Problem of Gradual Opening in Wave Rotor Passages." J. Propulsion, Vol. 1, No. 1, pp. 23-28, 1985.

3. Larosiliere, L. M. and Mawid, M.: "Analysis of Unsteady Wave Processes in a Rotating Channel." AIAA 93-2527.

4. Welch, G. E.: "Two-Dimensional Numerical Study of Wave Rotor Flow Dynamics." AIAA 932525.

5. Erdos, J. I., Alzner, E., and McNally, W.: "Numerical Solution of Periodic Transonic Flow Through a Fan Stage." AlAA J. 15 (1980), pp. 1559-1568.

6. Vavra, M. H.: Aero-Thermodynamics and Flow in Turbomachines. John Wiley \& Sons, Inc., 1960.

7. Chima, R. V. and Yokota, J. W.: "Numerical Analysis of Three-Dimensional Viscous Internal Flows." AIAA J., Vol. 28, No. 5, pp. 798-806, May 1990.

8. Thompson, K. E.: "Time-Dependent Boundary Conditions for Hyperbolic Systems, II." J. of Computational Physics 89, pp. 439-461 (1990). 9. Paxon, D. E. and Wilson, J.: "An Improved Numerical Model for Wave Rotor Design and Analysis." AIAA-93-0482. ( see also NASA TM105915). 


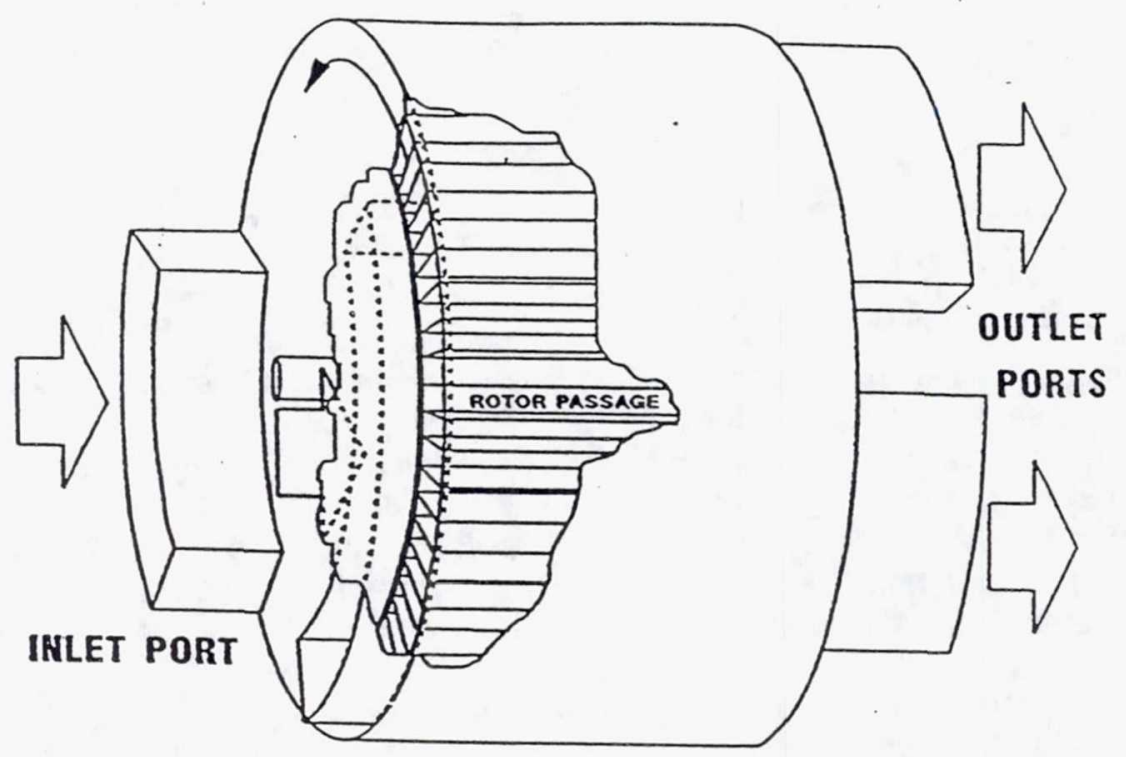

Figure 1. Wave Rotor Schematic Diagram.
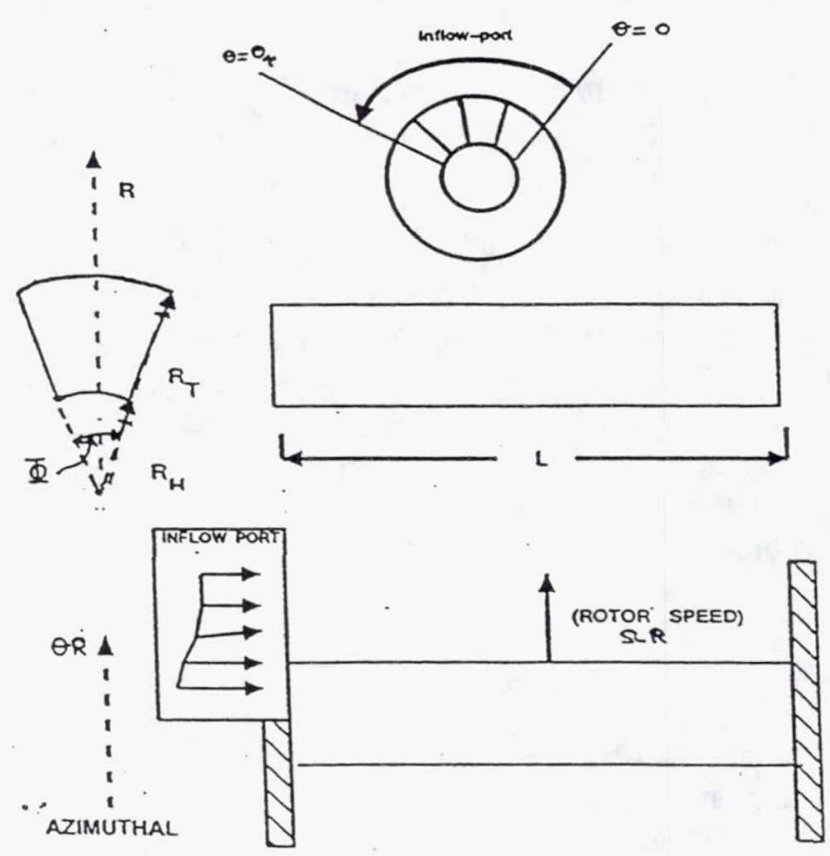

Figure 2. Geometric configuration for simulating the gradual opening process in a wave rotor. 

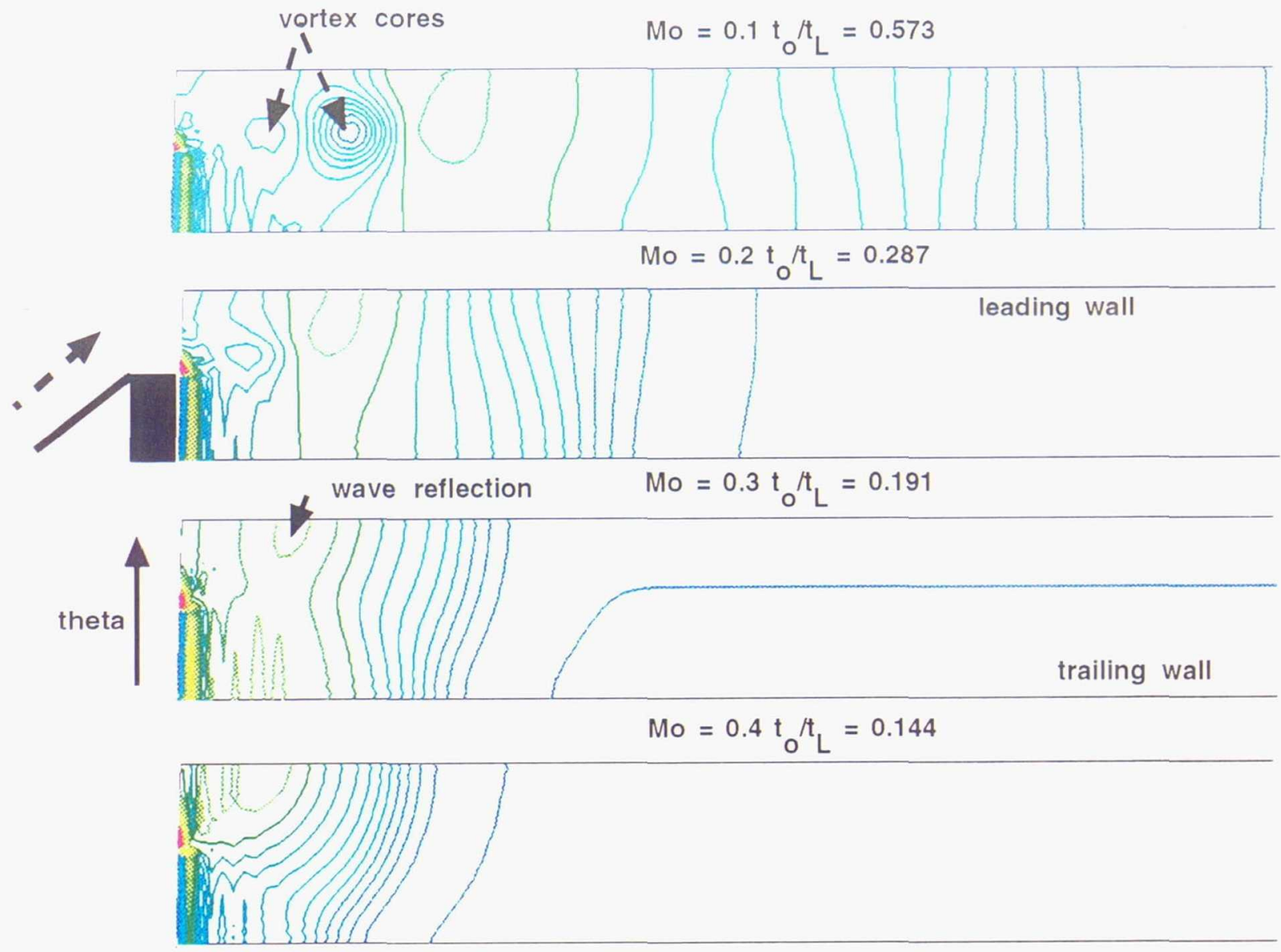

(a) half opened t $=0.5$
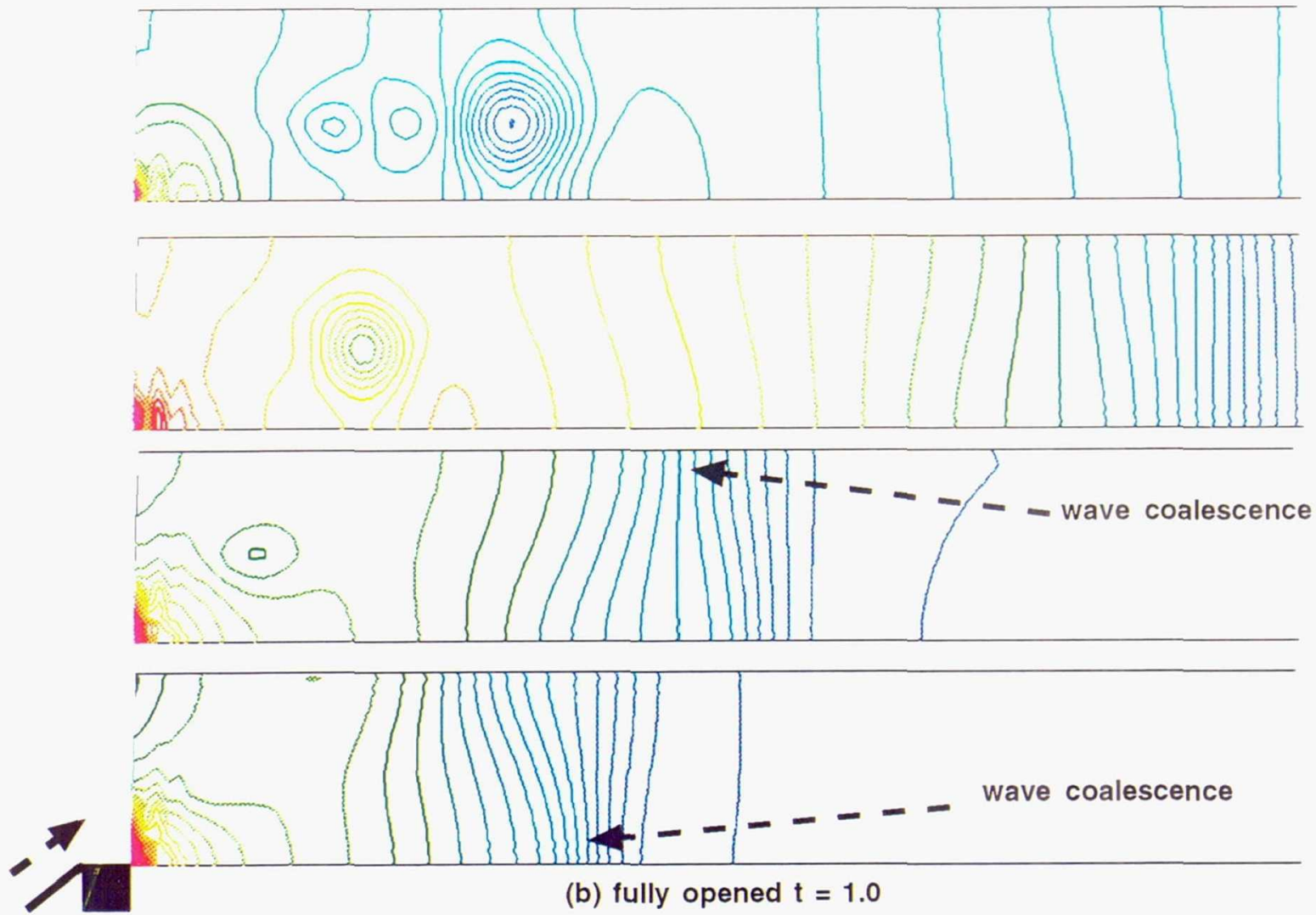

wave coalescence

Figure 3. Pressure contours in a passage-to-passage plane at mid-height illustrating the evolution of compression waves during the gradual opening transient for various wheel Mach numbers (Mo) and ratios of opening time to longitudinal transport time $\left(t_{0} / t_{L}\right)$. 
Page intentionally left blank 


$$
M o=0.1 t_{0} / t_{L}=0.573
$$

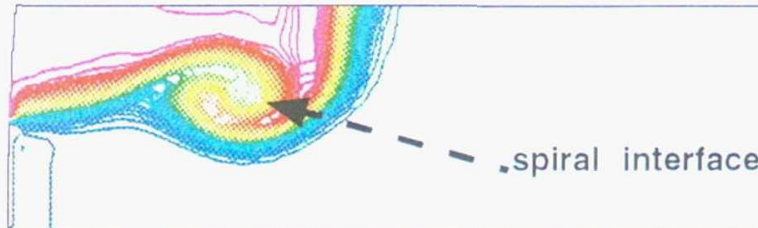

$$
\text { Mo }=0.2 t_{O} / t_{L}=0.287
$$

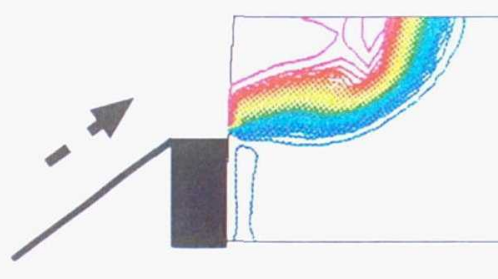

$$
\text { Mo }=0.3 t_{0} / t_{L}=0.191
$$

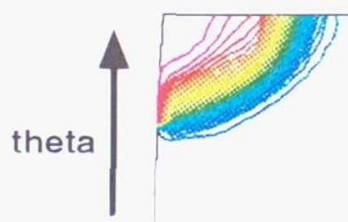

$$
\text { Mo }=0.4 t_{0} / t_{L}=0.144
$$

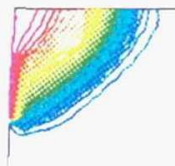

(a) half opened
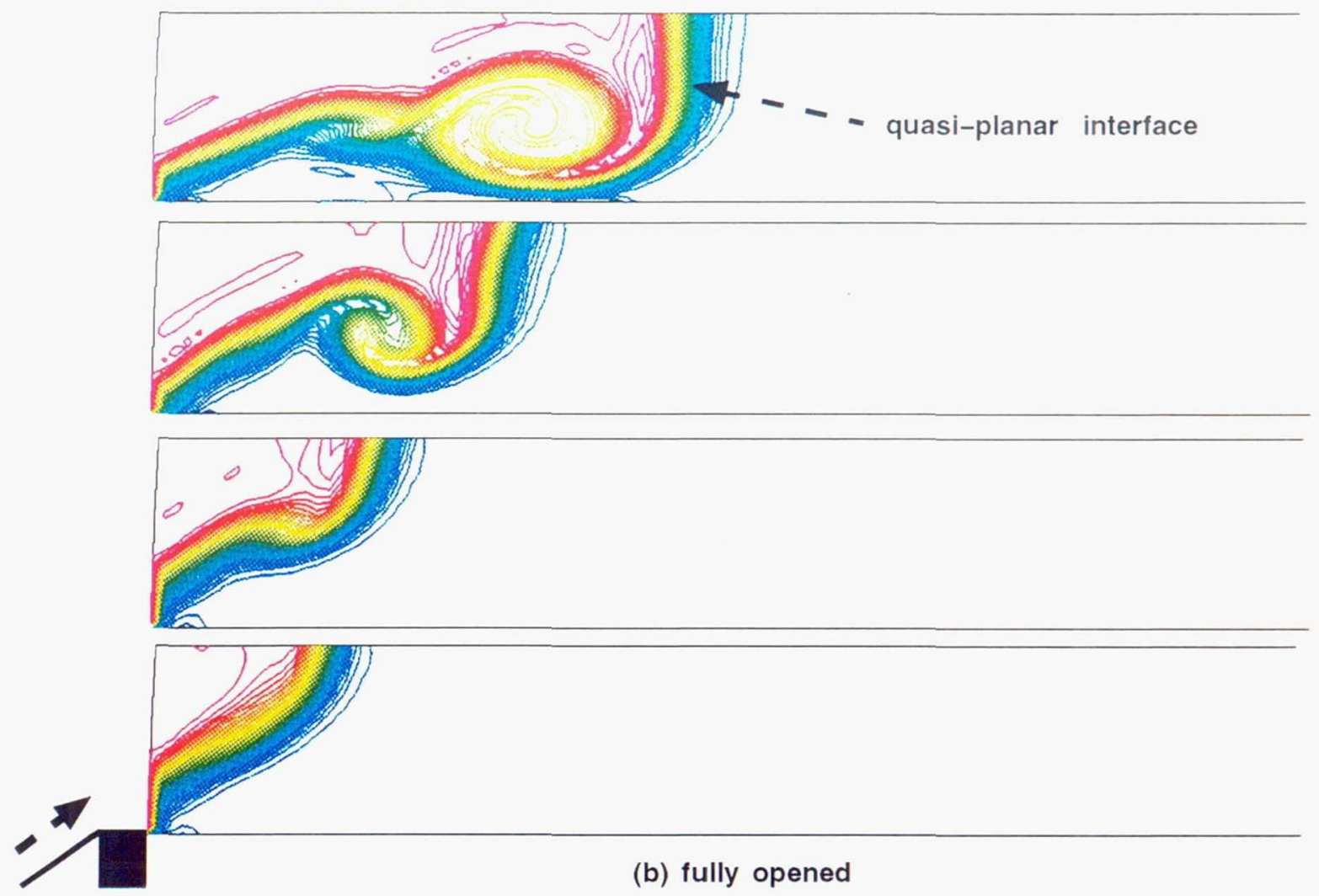

(b) fully opened

Figure 4. Evolution of the interface for different wheel Mach numbers (Mo) at two instants during the gradual opening transient. The contours correspond to entropy in a passage-to-passage plane at mid-height. 
Page intentionally left blank 


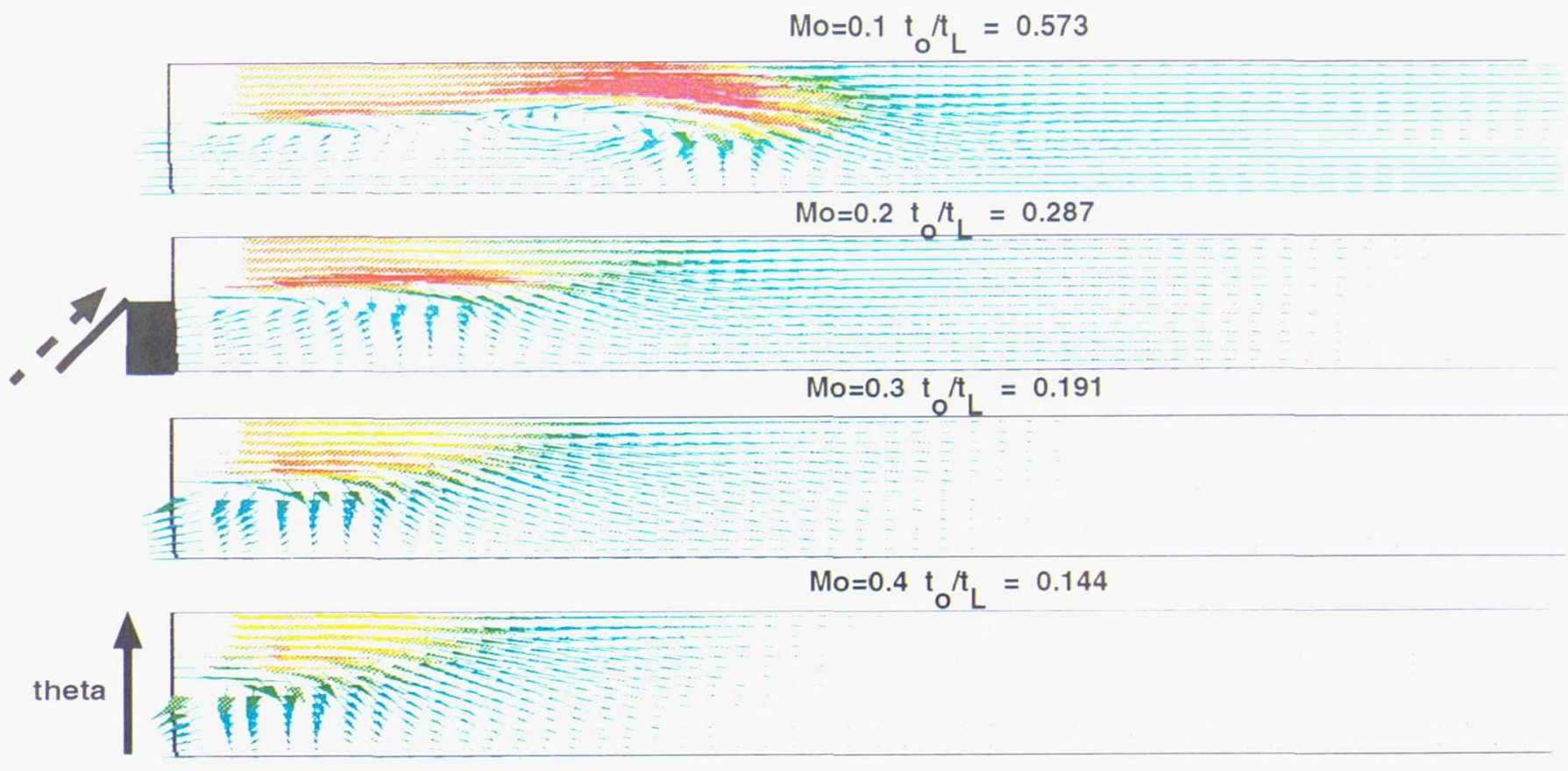

(a) half opened $t=0.5$
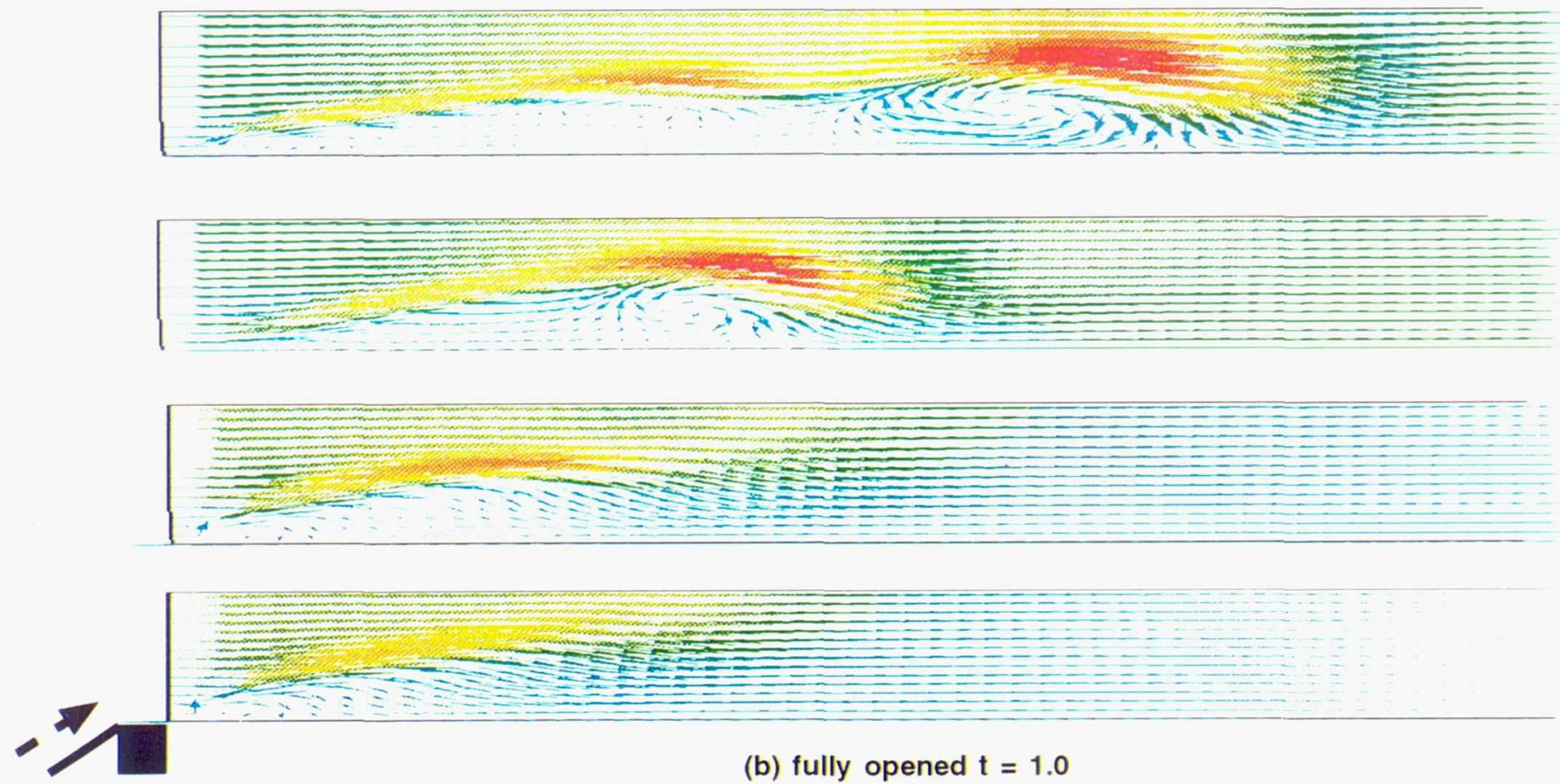

(b) fully opened t $=1.0$

Figure 5. Velocity vectors in a passage-to-passage plane at mid-height during the gradual opening transient 
Page intentionally left blank 


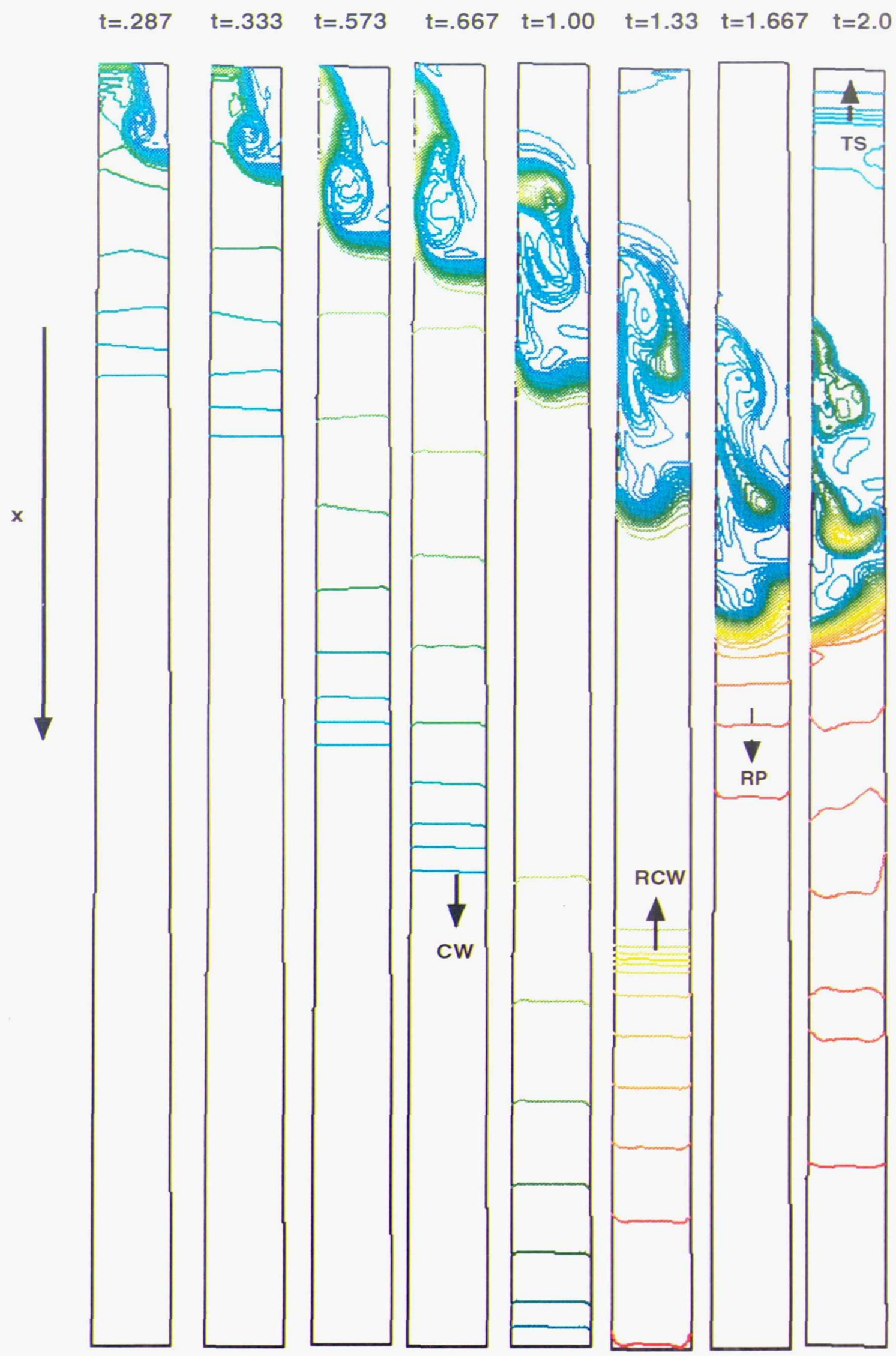

Figure 6(a). Density contours in a passage-to-passage plane at mid-height for a wheel Mach number Mo=0.1. $\mathrm{CW}$-compression wave; RCW-reflected compression wave; RP-reflected pressure wave; TS transmitted shock. 
Page intentionally left blank 


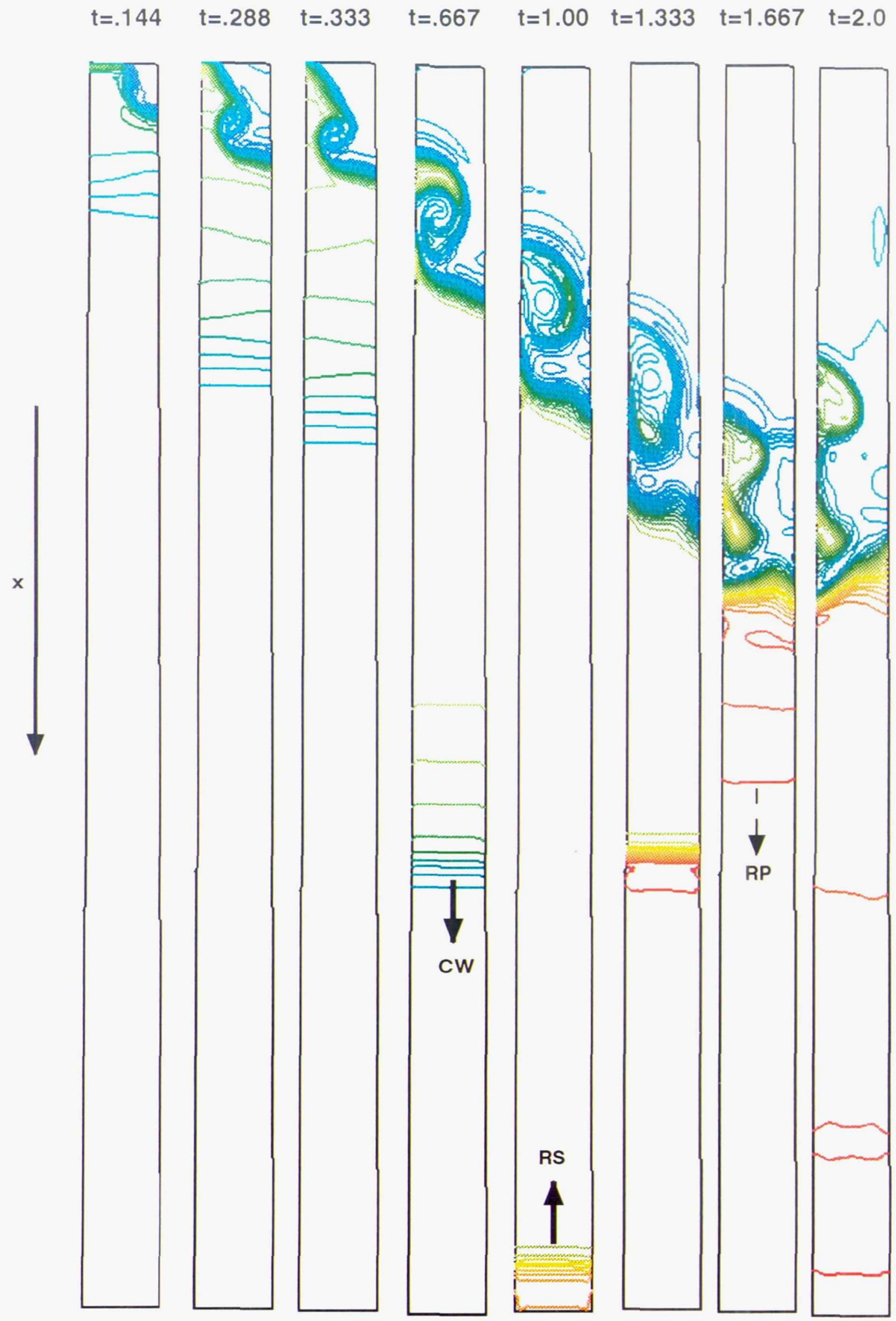

Figure 6(b) Density contours in a passage-to-passage plane at mid-height for a wheel Mach number Mo=0.2. $\mathrm{CW}$-compression wave; RS-reflected shock: RP-reflected pressure wave. 
Page intentionally left blank 


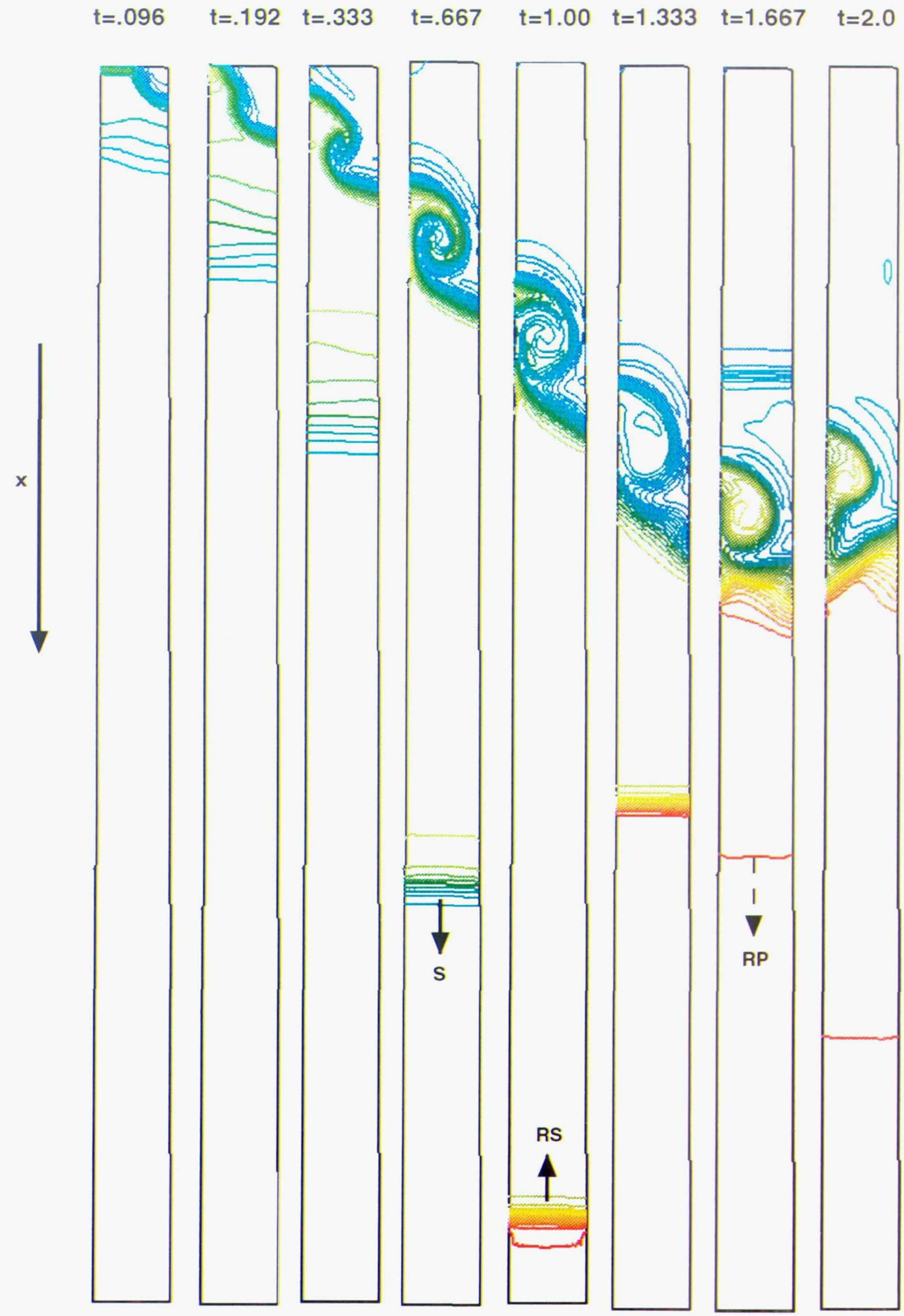

Fiigure 6(c). Density contours in a passage-to-passage plane at mid-height for a wheel Mach number Mo=0.3. S-shock; RS-reflected shock: RP-reflected pressure wave. 
Page intentionally left blank 


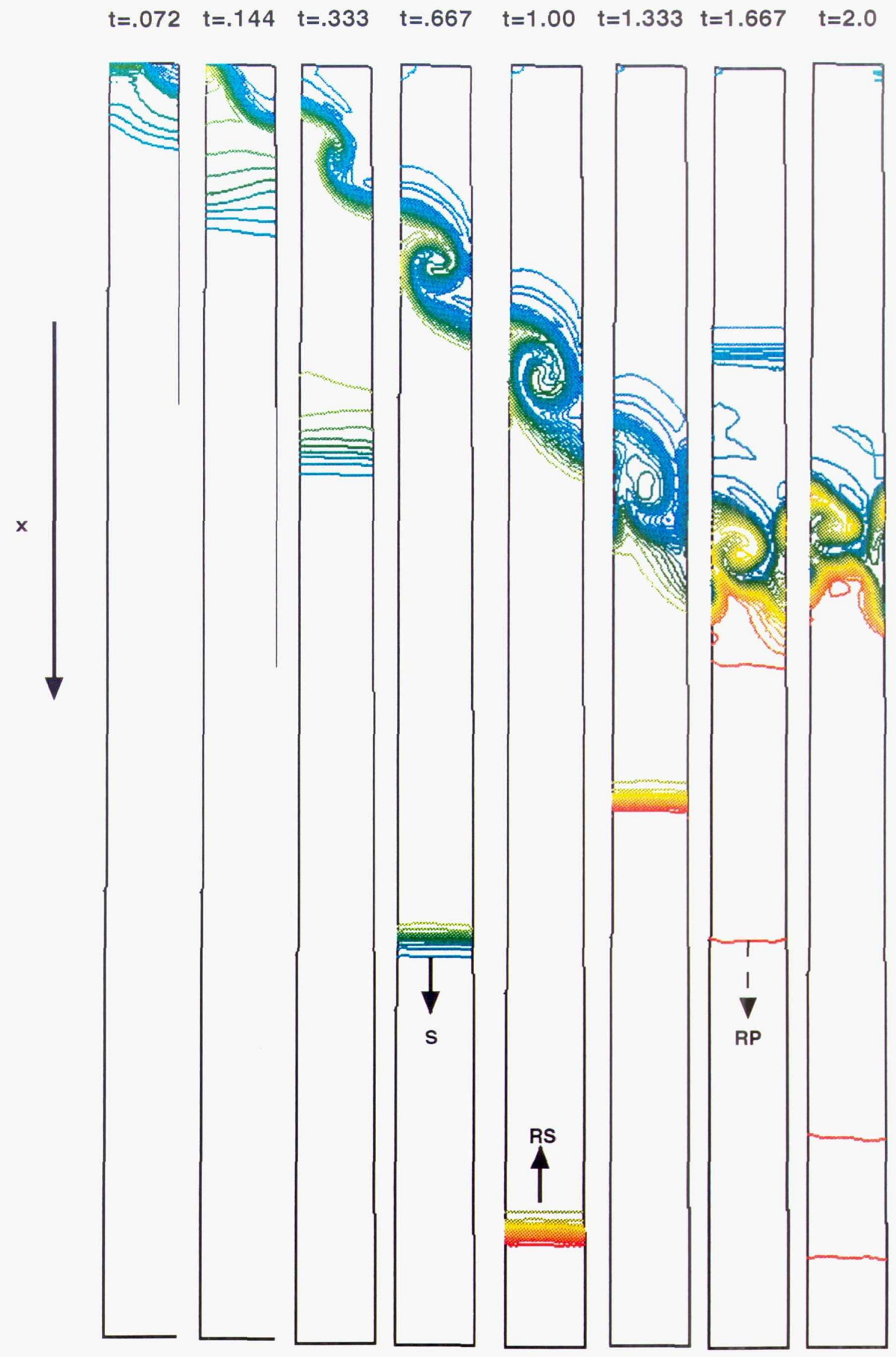

Figure 6(d). Density contours in a passage-to-passage plane at mid-height for a wheel Mach number Mo=0.4. S-shock; RS-reflected shock; RP-reflected pressure wave. 
Page intentionally left blank 


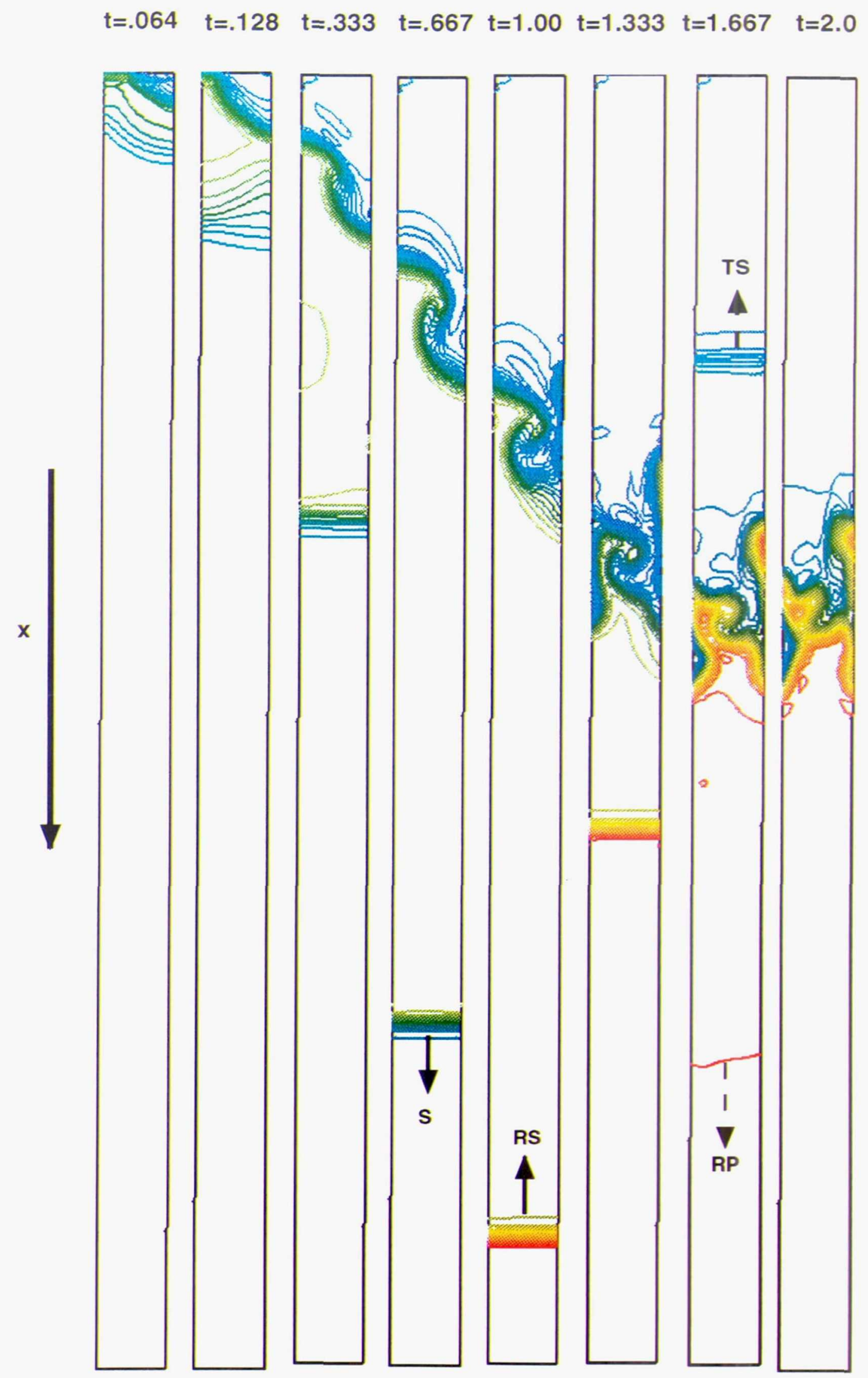

Figure 6(e). Density contours in a passage-to-passage plane at mid-height for a wheel Mach number Mo=0.6. S-shock; RS-reflected shock; RP-reflected pressure wave; TS-transmitted shock. 
Page intentionally left blank 

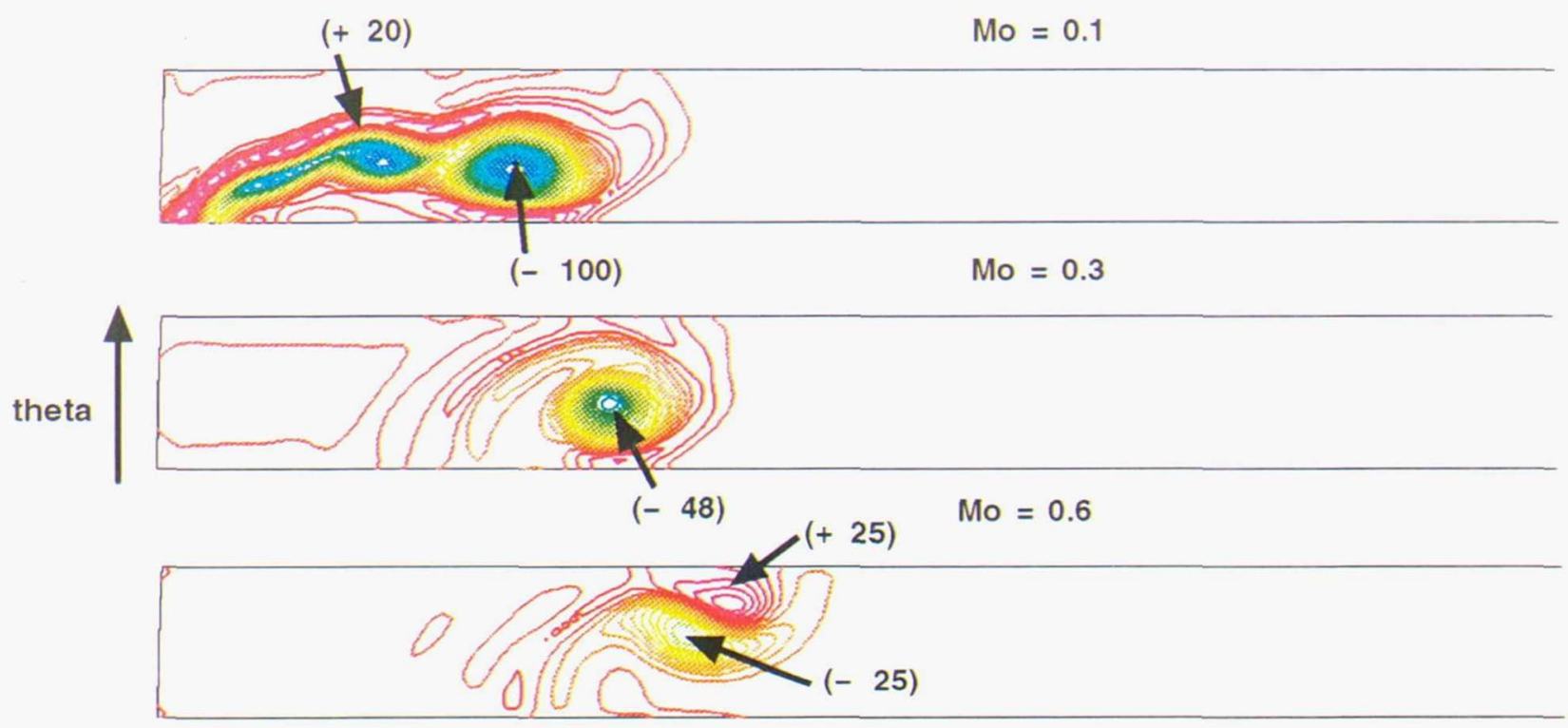

(a) $\mathrm{t}=0.667$
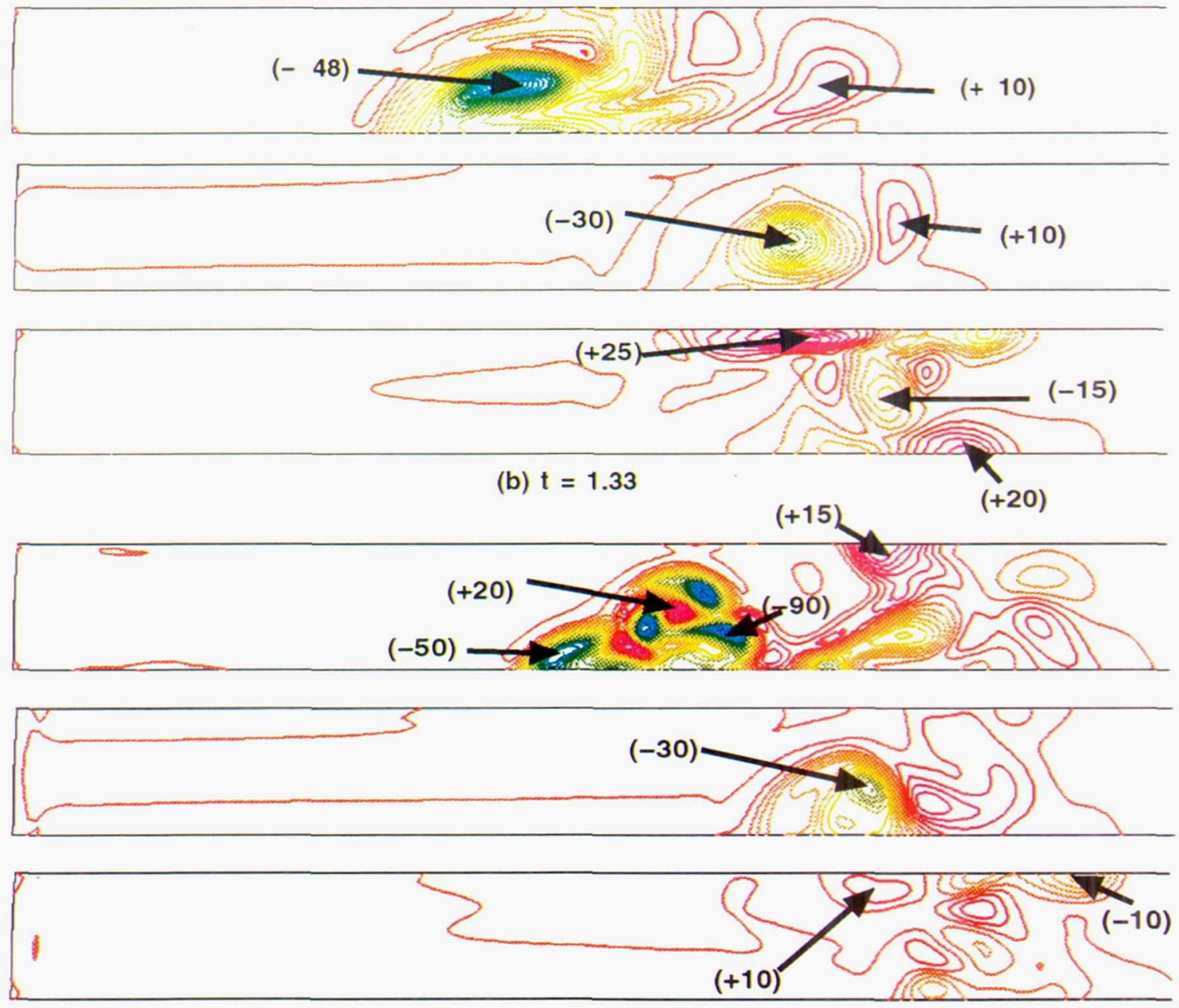

(c) $\mathrm{t}=2.0$

Figure 7. Contours of the radial component of vorticity in a passage-to-passage plane at mid-height. $(-)$ corresponds to a clockwise rotation; $(+)$ corresponds to a counter-clockwise rotation. 
Page intentionally left blank 


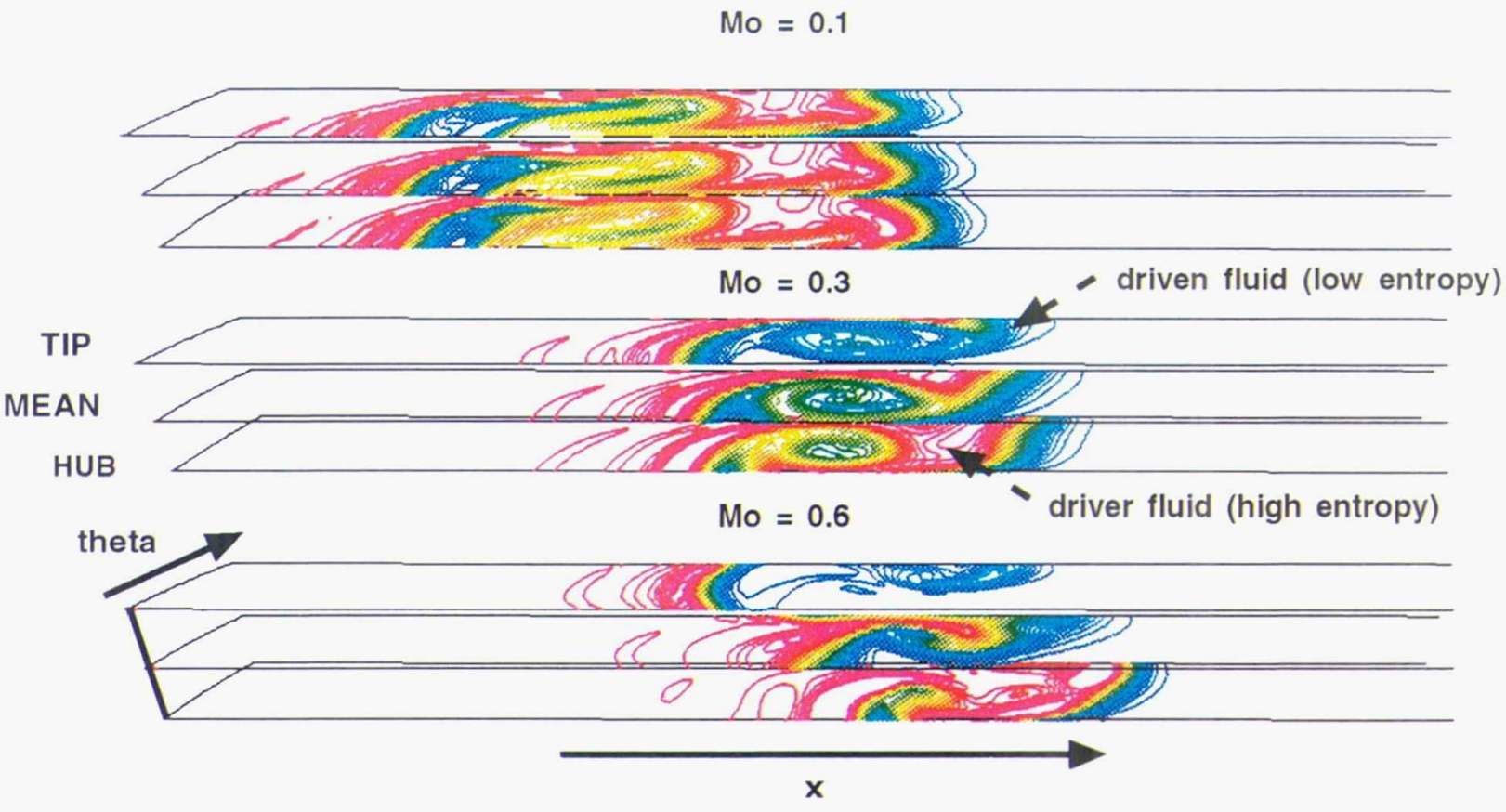

(a) $t=1.0$

spiral ,vortex
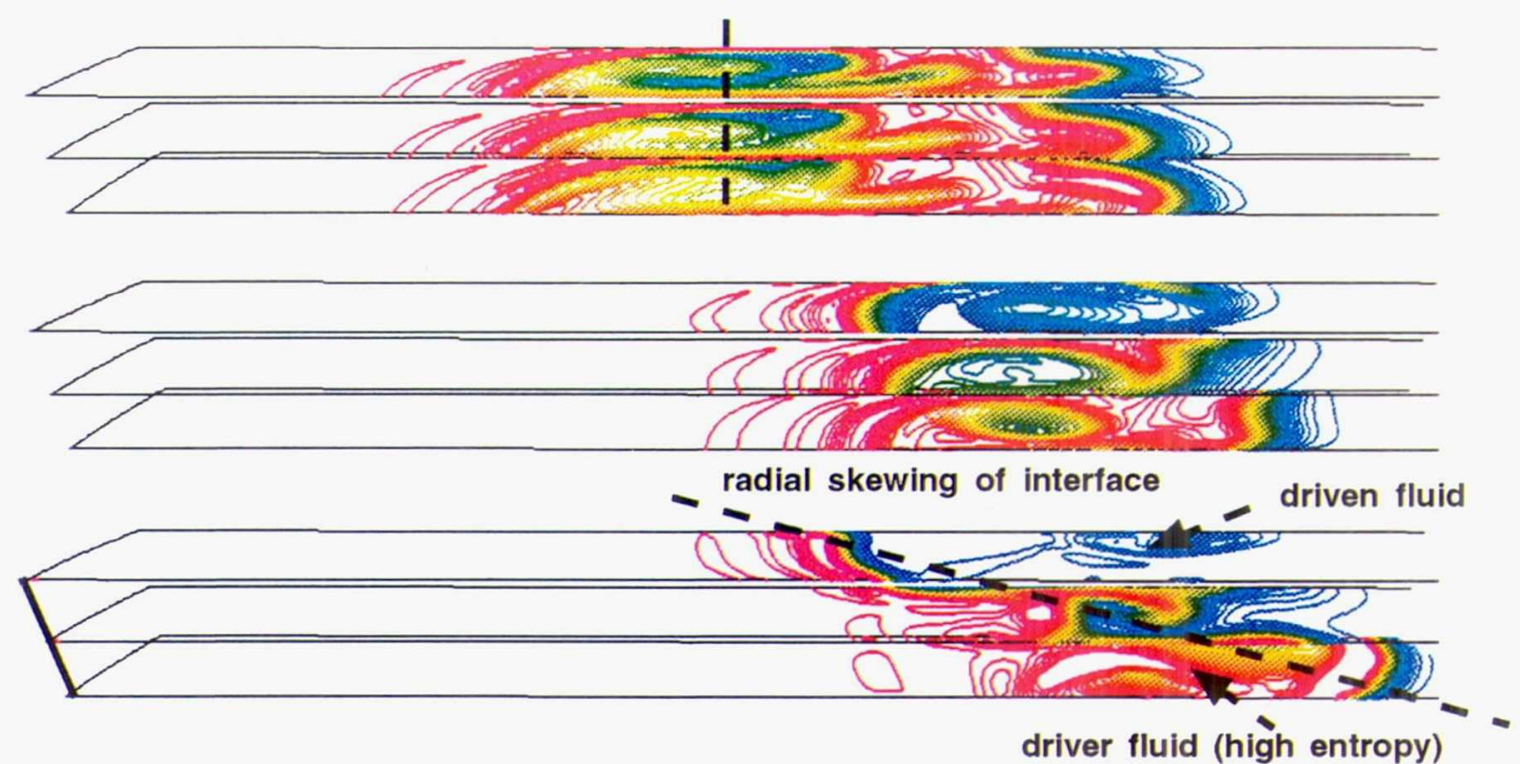

(b) $\mathrm{t}=1.33$

Figure 8. Entropy contours for various wheel Mach numbers at two instants subsequent to the gradual opening transient. The three-dimensionality of the interfacial structure is illustrated for $\mathrm{Mo}=0.3$ and 0.6 . 
Page intentionally left blank 


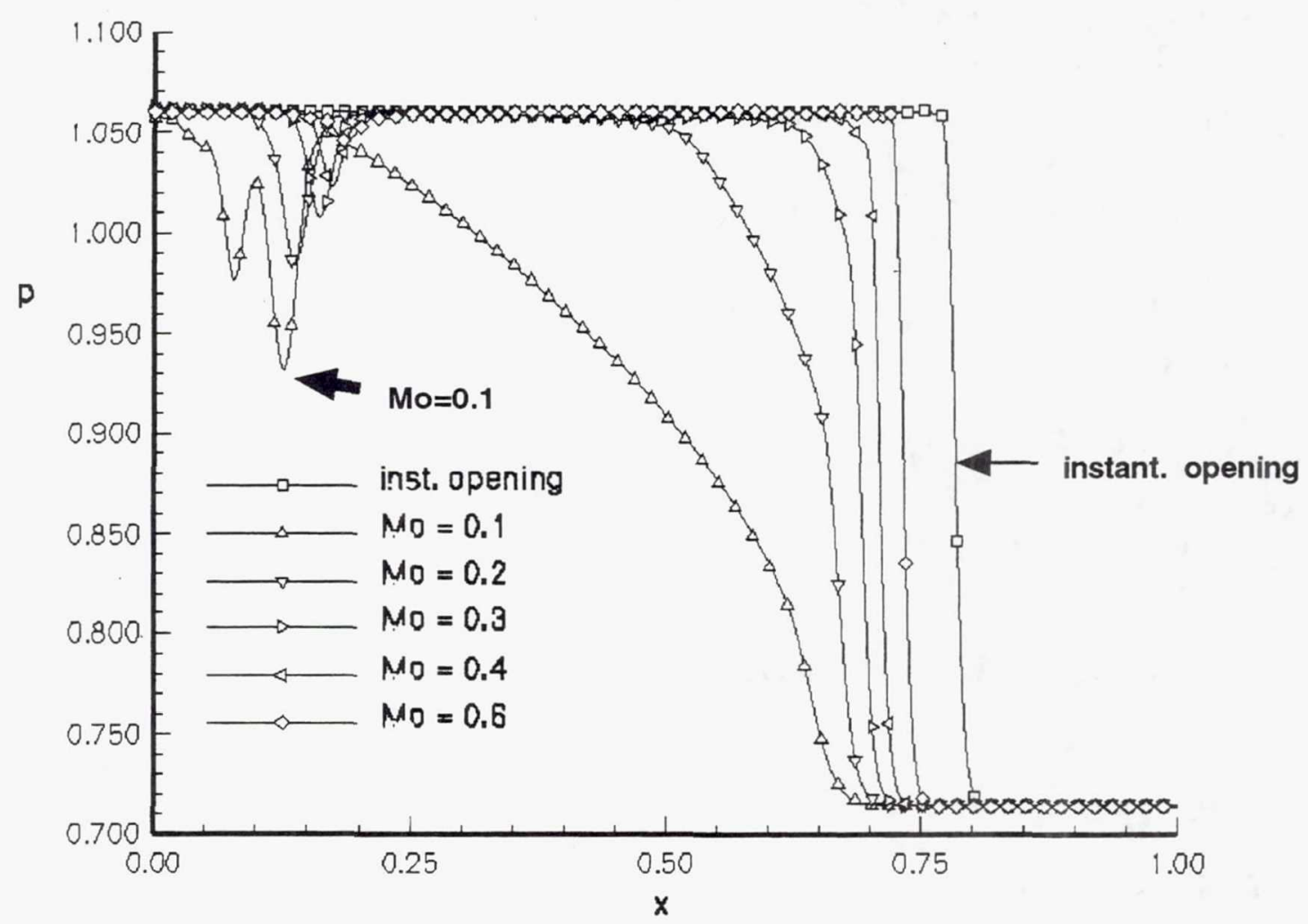

(a) static pressure

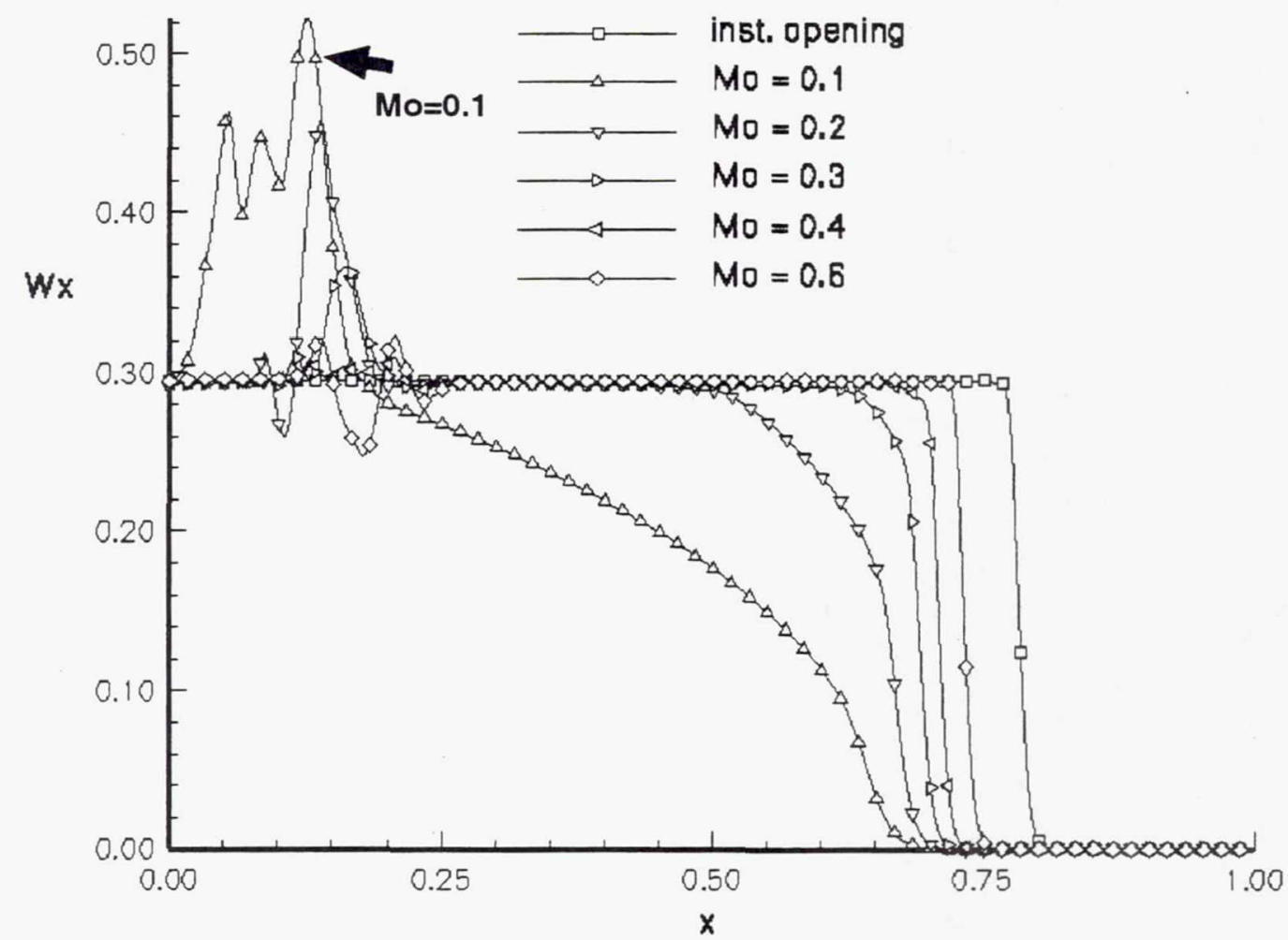

(b) axial velocity

Figure 9. Static pressure and axial velocity distribution along passage length at mid-height and mid-passage for $\mathrm{t}=0.667$. 
Public reporting burden for this collection of information is estimated to average 1 hour per response, including the time for reviewing instructions, searching existing data sources, gathering and maintaining the data needed, and completing and reviewing the collection of information. Send comments regarding this burden estimate or any other aspect of this collection of information, including suggestions for reducing this burden, to Washington Headquarters Services, Directorate for Information Operations and Reports, 1215 Jefferson Davis Highway, Suite 1204, Arlington, VA 22202-4302, and to the Office of Management and Budget, Paperwork Reduction Project (0704-0188), Washington, DC 20503.

\begin{tabular}{|l|c|c|}
\hline 1. AGENCY USE ONLY (Leave blank) & $\begin{array}{c}\text { 2. REPORT DATE } \\
\text { June } 1993\end{array}$ & $\begin{array}{r}\text { 3. REPORT TYPE AND DATES COVERED } \\
\text { Final Contractor Report }\end{array}$ \\
\hline
\end{tabular}

\section{TITLE AND SUBTITLE}

Three-Dimensional Numerical Simulation of Gradual

Opening in a Wave Rotor Passage

6. AUTHOR(S)

Louis M. Larosiliere
5. FUNDING NUMBERS

WU-505-62-10

C-NCC-208

7. PERFORMING ORGANIZATION NAME(S) AND ADDRESS(ES)

Ohio Aerospace Institute

22600 Cedar Point Road

Brook Park, Ohio 44142

8. PERFORMING ORGANIZATION REPORT NUMBER

E-7957

AIAA-93-2526

9. SPONSORING/MONITORING AGENCY NAME(S) AND ADDRESS(ES)

10. SPONSORING/MONITORING AGENCY REPORT NUMBER

National Aeronautics and Space Administration

Lewis Research Center

NASA CR-191157

Cleveland, Ohio 44135-3191

\section{SUPPLEMENTARY NOTES}

Prepared for the 29th Joint Propulsion Conference and Exhibit, cosponsored by the AIAA, SAE, ASME, and ASEE, Monterey, California, June 28-30, 1993. Project Manager, Lawrence C. Bober, Propulsion Systems Division, (216) 433-3944.

12a. DISTRIBUTION/AVAILABILITY STATEMENT

12b. DISTRIBUTION CODE

Unclassified - Unlimited

Subject Category 07

13. ABSTRACT (Maximum 200 words)

The evolution of the contact interface and the propagation of compression waves inside a single wave rotor passage gradually opening to and traversing an inlet port is studied numerically using an inviscid formulation of the governing equations. Insights into the response of the interface and kinematics of the flow field to various opening times are given. Since the opening time is inversely proportional to the rotational speed of the rotor, the effects of passage rotation such as centripetal and Coriolis accelerations are intrinsically coupled to the gradual opening process. Certain three-dimensional features associated with the gradual opening process as a result of centripetal and Coriolis accelerations are illustrated. For the range of opening times or rotational speeds considered, a portion of the interface behaves like a vortex sheet that can degenerate into a complex interfacial structure. The vortices produced along the interface can serve as a stirring mechanism to promote local mixing. Coriolis and centripetal accelerations can introduce threedimensional effects such as interfacial distortions in meridional planes and spanwise migration of fluid elements.

\begin{tabular}{|c|c|}
\hline \multicolumn{2}{|c|}{ Numerical simulation; Unsteady gas dynamics; Wave ro } \\
\hline $\begin{array}{l}\text { 17. SECURITY CLASSIFICATION } \\
\text { OF REPORT }\end{array}$ & $\begin{array}{l}\text { 18. SECURITY CLASSIFICATION } \\
\text { OF THIS PAGE }\end{array}$ \\
\hline Unclassified & Unclassified \\
\hline
\end{tabular}

\begin{tabular}{|l|l|}
\hline & $\begin{array}{c}\text { 15. NUMBER OF PAGES } \\
34\end{array}$ \\
\cline { 2 - 2 } & $\begin{array}{c}\text { 16. PRICE CODE } \\
\text { AO3 } 3\end{array}$ \\
\hline $\begin{array}{l}\text { 19. SECURTY CLASSIFICATION } \\
\text { OF ABSTRACT } \\
\text { Unclassified }\end{array}$ & 20. LIMITATION OF ABSTRACT \\
\hline
\end{tabular}


National Aeronautics and Space Administration

Lewis Research Center

Cleveland, Ohio 44135

Oriteial Dusinese

Peneity for Priverte Ues 8300
FOURTH CLASS MAIL

ADDRESS CORRECTION REQUESTED 